GLOBAL JOURNAL OF GEOLOGICAL SCIENCES VOL. 11, 2013: 13-26

\title{
PREMIÈRES DONNÉES MICROSTRUCTURALES SUR LE COMPLEXE GRANITO-MIGMATITIQUE DE LA RÉGION DE NIKKI, NE- BÉNIN
}

\author{
P. AFFATON., M. S. TAIROU., C. TOSSA., D. CHALA AND M. KWEKAM
}

(Received 29 April 2013; Revision Accepted 17 June 2013)

\begin{abstract}
RÉSUMÉ
L'ensemble lithostructural de Nikki, au NE-Bénin, appartient à la zone interne de la chaîne panafricaine des Dahomeyides. II correspond à un complexe réunissant des faciès d'orthogneiss granodioritiques, des migmatites et quelques intrusions granitiques associées. Les microstructures analysées, pour la première fois, dans ces ensembles lithologiques permettent de reconstruire cinq phases de déformation marquant la remobilisation panafricaine du bouclier bénino-nigérian. Cette tectogenèse débute avec le développement de la foliation $\mathrm{Sn}$ associée à la phase de collision $(\mathrm{Dn})$ entre le bouclier bénino-nigérian et la marge orientale du craton ouest-africain. La foliation principale $(S n+1)$ est attribuée à la phase majeure $D n+1$ qui induit la transposition du plan $S n$ et la refonte des paragenèses synDn dans les conditions du faciès amphibolite. La phase de déformation $D n+2$ est surtout matérialisée par le plan de cisaillement dextre $\mathrm{C} n+2$ assez pénétratif à l'échelle de l'affleurement. La phase de déformation $\mathrm{Dn}+3$ se traduit par la superposition d'un plan de cisaillement senestre $\mathrm{C} n+3$. Les marqueurs de déformation cassante composent deux systèmes de familles de décrochements attribués aux épisodes tardi-Dn+3 et syn-Dn+4. Ces systèmes de décrochements indiquent des axes de paléocontraintes de compression majeure respectivement ESE-WNW et SENW à SSE - NNW.
\end{abstract}

Mots clés : Bénin, Panafricain, Dahomeyides, zone interne, microstructures

\section{ABSTRACT}

Preliminary Microstructural Data on the Granito-Migmatitic Complex of Nikki region, NE-Benin

The Nikki lithostructural suite in northeastern Benin belongs to the internal zone of the Pan-African Dahomeyide belt .It corresponds to a complex composed of granodioritic orthogneiss and magmatites associated with a few granitic intrusions. The microstructures analyzed for the first time in this complex has led to the reconstruction of five deformation phases marking the Pan-African remobilization of the Benino-Nigerian shield. This tectogenesis begins with the development of the $\mathrm{Sn}$ foliation associated with the collision between the Benino-Nigerian shield and the eastern margin of the West African craton (Dn phase). The principal foliation $(S n+1)$ is attributed to the major $\mathrm{Dn}+1$ phase which led to the transposition of the $\mathrm{Sn}$ plane and recasting of the Sn-Dn paragenèses under amphibolites facies conditions. The $\mathrm{Dn}+2$ deformation phase is well expressed by the $\mathrm{Cn}+2$ dextral shear plane which is penetrative at outcrop scale. The $\mathrm{Dn}+3$ deformation phase is expressed by the superposition of a $\mathrm{Cn}+3$ sinistral shear plane. The markers of brittle deformation consist of families of strike-slip faults attributed to late $\mathrm{Dn}+3$ and syn$\mathrm{Dn}+4$ events. These systems of strike-slip faults indicate major compressive paleostress axes trending ESE-WSW and SE-NW to SSE-NNW respectively.

KEY WORDS: Benin, Pan-African, Dahomeyide, Internal Zone, Microstructures

\section{INTRODUCTION}

La présente analyse des marqueurs de tectogenèse panafricaine (600 $\pm 50 \mathrm{Ma})$ concerne précisément le complexe granito-migmatitique de la région de Nikki, au NE-Bénin (fig. 1). L'ensemble lithostructural de Nikki appartient aux unités internes de la chaîne des Dahomeyides (Affaton et al., 1991). Limité à l'Ouest par le complexe de la zone axiale de la "Faille de Kandi », il se distingue par des directions structurales NE-SW à ENE-WSW, non conformes à l'allure subméridienne des unités occidentales des Dahomeyides.

P. Affaton, CEREGE - UMR 7330 CNRS, Aix Marseille Université, BP 80 Europôle Méditerranéen de l'Arbois, 13545 Aix-En-Provence cedex 04, France

M. S. Tairou, Département de Géologie, Faculté des Sciences, Université de Lomé, BP 1515 Lomé, Togo

C. Tossa, Ministère des Recherches Pétrolières et Mines, 01 BP 249 OBRGM Cotonou, Bénin

D. Chala, Ministère des Recherches Pétrolières et Mines, 01 BP 249 OBRGM Cotonou, Bénin

M. Kwekam, Département des Sciences de la Terre, Université de Dschang, BP 67 Dschang, Cameroun 
A l'image de tout le bouclier bénino-nigérian, les caractéristiques structurales du complexe de Nikki restent non définies ou très sommairement connues (Affaton et al., 1978; 1991). En particulier, les différentes phases de déformation enregistrées ne sont pas identifiées et caractérisées comme dans les unités frontales occidentales (Affaton, 1990; Tairou, 2006 ; Tairou et al., 2007). Des relevés de microstructures récemment effectués dans le Nord-Bénin permettent d'amorcer l'identification des marqueurs de tectogenèse au sein du métacraton bénino-nigérian. Cette contribution préliminaire rentre ainsi dans le cadre de la recherche du modèle d'implication de ce métacraton dans l'édification de l'orogène des Dahomeyides.

\section{Cadre géologique}

La chaîne panafricaine des Dahomeyides (fig. 1) résulte d'un long processus de convergence ayant abouti à la collision entre le bouclier bénino-nigérian et la marge sud-orientale du craton ouest-africain, à la fin du Néoprotérozoïque (Caby, 1989 ; Affaton et al., 1991 ; Attoh et al., 1997). Ce segment méridional de l'orogène transsaharien comprend, dans sa partie frontale, un chapelet subméridien de massifs basiques à ultrabasiques matérialisant la zone de suture (fig. 1). Ce chapelet délimite la zone externe, à l'Ouest, et la zone interne, à l'Est.

Les unités de la zone externe des Dahomeyides sont structurées en piles de nappes et d'écailles charriées vers l'Ouest, sur le bassin des Volta (Simpara et al., 1985 ; Affaton et al., 1991; Tairou, 2006). Les nappes les plus externes sont constituées de métasédiments variés (grès-quartzites, hématitites, métasilexites, schistes, métaconglomérats et métadiamictites) qui sont les équivalents latéraux et tectono-métamorphiques des mégaséquences inférieure et moyenne du bassin des Volta (Simpara, 1978; Affaton et al., 1980). Ce sont ces nappes qui composent les unités structurales du Buem et de l'Atacora (fig. 1) et supportent tectoniquement les nappes des unités orthogneissiques externes (unité de Kara-Niamtougou; unité de Kpalimé-Amlamé ou Ho ; Tairou et al., 2009 ; Agbossoumondé et al., 2007). Ces dernières sont considérées comme des témoins du substratum éburnéen (2000 $\pm 200 \mathrm{Ma}$ ) largement remobilisé par la thermo-tectonique panafricaine (Caen-Vachette et al., 1979 ; Kalsbeek et al., 2012).

Les massifs de la zone de suture (dont les plus connus sont ceux de Dérouvarou, Kabyè-Kpaza, Djabatouré-Anié, Agou-Ahito et Shai ou Akuse; fig. 1) sont identifiés comme des ensembles de nappes éclogitiques à granulitiques charriées sur les unités externes (Tairou, 2006 ; Sabi, 2007). Ils sont constitués de granulites variées et parfois d'éclogitoïdes, associées à des métasédiments (micaschistes, quartzites et gneiss à grenat et disthène), des lambeaux de pyroxénites et de carbonatites, et à des équivalents rétromorphiques (amphibolites, talcschistes et serpentinites; Agbossoumondé, 1998; Attoh et Nude, 2008). Ces massifs représentent des témoins du processus subduction-collision dans les Dahomeyides (640 - 610 Ma ; Affaton et al., 2000 ; Agbossoumondé et al., 2004 ; Ducleaux et al., 2006 ; Attoh et al., 2007).

La zone interne des Dahomeyides correspond à la portion méridionale du métacraton transsaharien (Abdelsalam et al., 2002). II s'agit de l'ensemble sur lequel s'établit la vaste pénéplaine dite béninonigériane. Son front occidental chevauchant est grossièrement délimité par le chapelet de massifs de la zone de suture (fig. 1). Cette portion de métacraton est constituée d'un complexe de base gneissomigmatitique, portant des ceintures métavolcanosédimentaires et plus ou moins envahi par des granitoïdes d'âge panafricain (Caen-Vachette, 1975 ; Rahaman et al., 1988 ; Affaton et al., 1991). A l'image de son équivalent septentrional (Hoggar central et oriental), le métacraton bénino-nigérian comporte de nombreux couloirs majeurs de cisaillement généralement subméridiens. Ces couloirs tel que celui de la "Faille de Kandi » (fig. 1), ont joué un rôle déterminant dans le processus de structuration des Dahomeyides (Ngako, 1999 ; Tairou et al., 2007).

Les études structurales des unités frontales des Dahomeyides, ont mis en évidence cinq phases de tectogenèse panafricaine dénommées $\mathrm{Dn}$ à $\mathrm{Dn}+4$ (Affaton, 1990; Tairou, 2006). La phase Dn serait pénécontemporaine de la collision entre la plaque chevauchante du bouclier bénino-nigérian et la bordure orientale du craton ouest-africain. Elle est matérialisée par la foliation $\mathrm{Sn}$ et datée de 612,5 \pm 0,8 Ma dans les granulites du Massif Kabyè (Affaton et al., 2000). La déformation $\mathrm{Dn+1}$ est considérée comme une phase de structuration majeure, avec la mise en place des nappes à vergence Ouest. Elle se traduit par une rétromorphose soulignée par les paragenèses de la foliation principale ou régionale $(S n+1)$. A cette phase tangentielle $D n+1$ se sont superposées trois phases de plissement postnappe $(D n+2$ à $D n+4)$. Ces derniers plissements sont responsables de l'implication de la foliation $\mathrm{Sn+1}$ dans des structures antiformes et synformes bien caractérisées au sein de l'unité structurale de l'Atacora (Tairou, 2006). 


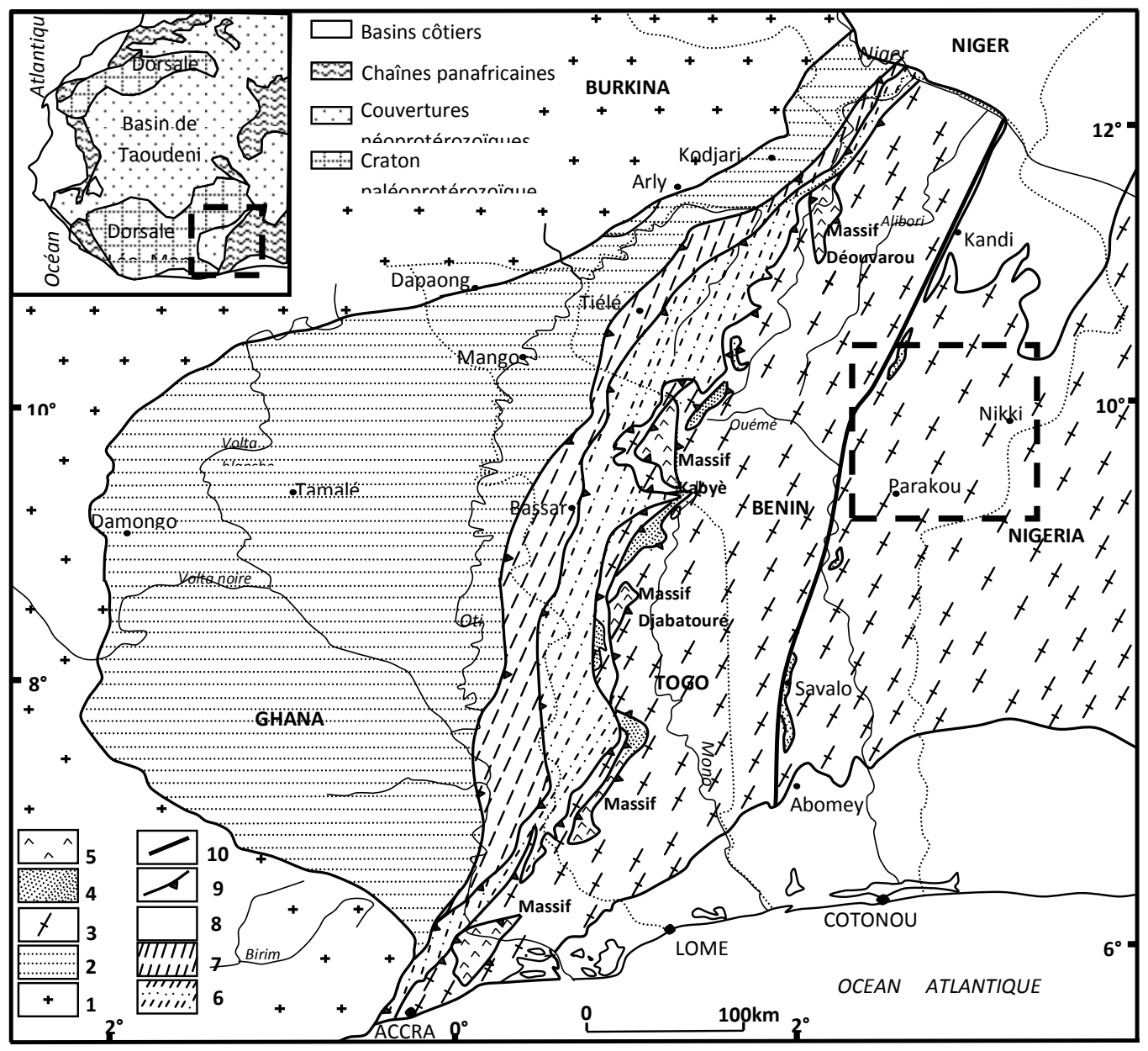

Fig. 1: Cadre géologique régional incluant le secteur de Nikki. 1 = substratum éburnéen de la dorsale de Léo-Man ; 2 $=$ couverture néoprotérozoïque du bassin des Volta ; $3=$ unités gneisso-migmatitiques internes et externes ; $4=$ quartzites micacés à disthène ; $5=$ massifs basiques et ultrabasiques de la zone de suture ; $6=$ Unité structurale de l'Atacora ; 7 = Unité structurale du Buem ; 8 = bassins paléozoïques à cénozoïques de Kandi et du golfe de Guinée ; $9=$ contact de charriage $; 10$ = zone mylonitique de la « Faille de Kandi ». 
Caractéristiques lithologiques du complexe de Nikki

Sur le plan lithologique, le complexe de Nikki peut être considérée comme une portion de socle gneisso-migmatitique associant quelques intrusions granitiques. Le complexe est charrié vers l'Ouest, sur l'ensemble de la zone axiale de la " Faille de Kandi » et s'ouvre vers l'Est sur le territoire nigérian (fig. 2).

Dans son ensemble, le complexe de Nikki est constitué de faciès variés d'orthogneiss et de migmatites. Ses orthogneiss sont mésocrates, à structure très orientée, avec des porphyroblastes millimétriques à centimétriques étirés dans la foliation. Ils ont une composition granitique à granodioritique, avec essentiellement du quartz, des feldspaths, de la biotite et parfois de l'amphibole. Ces roches présentent de rares enclaves biotitiques nettement transposées dans la foliation $\mathrm{Sn}+1$. Elles sont aussi associées à des métapegmatites parfois assez particulières, avec une structure orbiculaire définie par des porphyroblastes centimétriques de feldspaths orientés NE-SW au sein d'une matrice fine et plus ou moins abondante (fig. 3a). Ces porphyroblastes portent souvent une large couronne de recristallisation (fig. 3b). D'autres métapegmatites correspondent à des anciens filons généralement NE-SW (N40 à N55) et montrent plutôt une structure cataclastique, avec des cristaux de quartz et feldspaths fracturés.

A l'échelle microscopique, les orthogneiss sont caractérisés par une texture à nombreux porphyroclastes de microcline, de plagioclases et de quartz enrobés dans un fond granoblastique très orienté, peu abondant et essentiellement à quartz, plagioclases et biotite (fig. 3c). Cette dernière semble souligner la foliation principale $(S n+1)$, mais elle se retrouve aussi dans le plan de cisaillement dextre $(\mathrm{C} n+2)$. Elle est accompagnée de minéraux d'apparence post-tectonique que sont le sphène, la pistachite, la muscovite et la magnétite (fig. 3d). La hornblende verte apparaît en reliques dans la foliation principale $(S n+1)$ et recouverte de biotite (fig. $3 d$ ).

Les affleurements migmatitiques sont caractérisés par une grande hétérogénéité qui consiste en une association d'orthogneiss méso- à mélanocrates et de métagranites leucocrates (fig. 4a). L'ensemble est parcouru par des filons granitiques, aplitiques ou pegmatitiques plus ou moins tardifs (fig. 4b). Dans toutes ces roches, l'orientation minérale indique que la différenciation des faciès est antérieure au développement de la foliation principale $S n+1$. Les métagranites sont de couleur gris clair à rose et de composition essentiellement quartzo-feldspathique. Comme les faciès sombres, ils sont nettement orientés et contiennent de petites enclaves lenticulaires d'orthogneiss.

Certains affleurements sont constitués par de véritables anatexites. Ils sont assez homogènes et composés de leucogranites à grain moyen ou grossier, peu orientés et associés à de rares enclaves sombres.

En lame mince, les faciès granodioritiques sont comparables aux orthogneiss mentionnés plus haut. Dans les faciès les plus micacés, la biotite domine la hornblende verte et souligne la superposition d'une schistosité de flux $(\mathrm{Sn}+2)$ à la foliation principale (fig. 4c). Une abondante recristallisation de porphyroblastes subautomorphes de pistachite, zoïsite, muscovite, sphène et magnétite caractérise aussi ces orthogneiss. II s'agit de minéraux de recristallisation post-tectonique marquant un épisode de rétromorphose tardive.

Les intrusions granitiques s'individualisent comme de vastes plutons dont les affleurements sont massifs ou souvent chaotiques et renferment des enclaves décimétriques à métriques et de véritables septa de gneiss ou de migmatites. Ils sont traversés par de nombreux filons ou fentes de génération et d'orientation variées. Ces intrusions se composent de leucogranites à structure orientée ou équante. A première vue, on peut ainsi distinguer: les granites orientés ou tardi-tectoniques (type granite de Parakou) et les granites non orientés ou granites «posttectoniques " (type granite de Serikali). Le premier est d'un blanc plus ou moins moucheté. II montre de nombreux porphyroblastes millimétriques à centimétriques de feldspaths orientés dans les directions NNE-SSW (N15) à NE-SW (N60). L'orientation des cristaux de feldspath est localement associée à une rotation antihoraire liée au développement d'un plan de cisaillement senestre $(\mathrm{Cn}+3)$ subméridien à NE-SW (fig. $4 \mathrm{~d}$ ). Ce plan apparaît souvent souligné par une recristallisation de biotite. Les faciès de granite non orienté sont gris clair à roses, à grain fin à grossier et particulièrement riches en feldspath potassique.

\section{Tectogenèse panafricaine dans le complexe de Nikki}

Les éléments structuraux relevés dans le secteur de Nikki sont des marqueurs de déformation ductile ou cassante matérialisant cinq phases de tectogenèse panafricaine. On y distingue nettement :

- un plan $S n$, dû à la phase Dn et qui se retrouve généralement masqué ou oblitéré par transposition dans la foliation principale $\mathrm{Sn}+1$; une foliation $S n+1$, responsable de la structuration de l'ensemble des faciès; cette forte empreinte, attribuée à la phase tangentielle $\mathrm{Dn}+1$, est associée à des linéations minérales ou d'étirement Ln+1;

- une foliation ou schistosité de flux $S n+2$, associée aux plis $\mathrm{Pn}+2$ et qui est syngénétique d'un plan pénétratif de cisaillement dextre $(C n+2)$; ces marqueurs sont attribués à la phase de déformation $\mathrm{Dn}+2$;

- un plan de cisaillement senestre $(C n+3)$, syn$\mathrm{Dn}+3$ et généralement non pénétratif ;

- des plans de fractures tardives, correspondant généralement à des décrochements et probablement liés aux phases panafricaines tardives $(D n+3$ et $D n+4)$

\section{Marqueurs de déformation ductile}

La foliation $\mathrm{Sn+1}$ est omniprésente dans tous les faciès rocheux à l'exception des filons et intrusions granitiques tardifs. Elle confère aux roches tous leurs aspects architecturaux. Ce plan se définit dans les directions NE-SW (N55 à N65) à E-W (N80 à N95), avec des pendages assez variables (fig. 5). II porte une linéation minérale ou d'étirement $\mathrm{Ln}+1$, NNE-SSW à NE-SW et subhorizontale ou plongeant faiblement vers 
le Sud ou le Sud-Ouest (fig. 5). Le plan principal $\mathrm{Sn+1}$ correspond à une forte empreinte superposée à une foliation antérieure $(\mathrm{Sn})$ qui se dessine discrètement dans les enclaves et boudins de gneiss comme un plan nettement transposé ou légèrement discordant. II est aussi repris par la phase subséquente $D n+2$. Cette reprise est peu matérialisée par des plis $\mathrm{Pn}+2$. De fait, la déformation $\mathrm{Dn}+2$ est surtout soulignée par le plan de cisaillement dextre $\mathrm{Cn}+2$ qui apparaît pénétratif dans certains affleurements d'orthogneiss, comme celui de Pèrèrè (N09 $47^{\prime} 48.0^{\prime \prime}$ - E02 $\left.{ }^{\circ} 59^{\prime} 46.5^{\prime \prime}\right)$. Généralement, ce plan se retrouve dans des directions NE-SW (N40 à N65) et sa régularité confère parfois aux orthogneiss un débit en microlithons d'épaisseur centimétrique, avec des microplis d'entraînement à axes subverticaux ou à fort plongement sud-ouest (fig. 5 et 6a).

Un régime de cisaillement senestre syn-Dn+3 se retrouve remarquablement inscrit dans les ensembles lithologiques. II est matérialisé par le plan $\mathrm{Cn}+3$ subméridien (N10 à N25). Contrairement au plan $\mathrm{Cn}+2$, l'empreinte du cisaillement tardif $(\mathrm{Cn}+3)$ est irrégulière et non pénétratif à l'échelle de l'affleurement. Elle se distingue comme de petites zones d'épaisseur centimétrique et soulignées par une mylonite grise (fig. $6 b$ et $c$ ) et superposées aux structures antérieures. Les plans $\mathrm{Cn}+3$ sont parfois associés à des remplissages de quartz plus ou moins déformés par le mouvement cisaillant. Enfin, il convient d'attribuer à la phase $D n+3$ les structures synformes et antiformes dont les traces axiales NE-SW à ENE-WSW sont dessinées dans la synthèse cartographique d'Affaton et al. (1978).

\section{Marqueurs de déformation cassante}

Les matériaux du secteur de Nikki portent aussi des marques d'une tectonique cassante panafricaine matérialisée par de nombreux plans de décrochement. L'analyse chronologique permet de distinguer essentiellement deux générations de fractures comparables à celles attribuées aux phases tardives de tectogenèse panafricaine dans les Dahomeyides au Nord-Togo et au SE-Ghana (Tairou et al., 2007 ; 2012). Dans le cadre de la présente analyse préliminaire, environ 200 plans de décrochement conjugués ont été relevés sur 5 sites faillés (fig. 2). Après un dépouillement stéréographique manuel de ces plans, complétant la caractérisation géométrique des stries, l'analyse des familles de décrochement conjuguées, par la méthode informatique de calcul de tenseur de contraintes (Angelier, 1990) a permis d'obtenir, pour une première fois, des indications d'axes de paléocontraintes panafricaines dans le Nord-Bénin (tabl. 1).

\section{Les décrochements syn-Dn+3}

Les miroirs des décrochements les plus anciens, probablement dus à la phase de déformation $\mathrm{Dn}+3$, sont généralement recouverts d'un tapis de recristallisation souvent biotitique auquel se superpose une nette striation subhorizontale (fig. $6 \mathrm{~d}$ et e). Le tapis de biotite apparaît parfois verdâtre par chloritisation secondaire. Ce type de plans subverticaux compose un système de deux familles de décrochement conjuguées (fig. $7 a$ et b), avec des plans dextres qui se retrouvent dans les directions NE-SW à E-W (N65 à N90) et des plans senestres définis dans les directions SE-NW
(N120 à N145). Ces familles de décrochement sont indicateurs d'une compression à axe principal $\sigma 1$ subhorizontal et de direction N100 à N110 (tabl. 1 et fig. $7 a$ et $b)$.

\section{Les décrochements tardifs ou syn-Dn+4}

Les fractures de la génération $D n+4$ semblent plus représentées sur les sites faillés étudiés. Leurs miroirs sont caractérisés par une recristallisation peu abondante. Cette dernière réunit, en particulier sur les miroirs développés dans les granites, du mica blanc et du quartz accompagnés de chlorite et de minéraux fibreux. Ce système de décrochement apparemment récent compose des plans $\mathrm{N} 100$ à $\mathrm{N} 120$ dextres et N160 à N190 senestres. L'analyse de ce système donne un tenseur de contrainte de compression, avec $\sigma 1$ subhorizontal et de direction SE-NW à SSE-NNW (N140 à $\mathrm{N} 160$; tabl. 1 et fig. $7 \mathrm{c}$ et d).

\section{Evolution tectono-métamorphique}

Les ensembles pétrographiques du complexe de Nikki témoignent d'une remobilisation générale panafricaine: celle-ci consiste en une large migmatisation accompagnée d'une tectogenèse en cinq phases. A l'échelle de l'affleurement, il est évident que la migmatisation est antérieure au développement de la foliation principale $(S n+1)$. Le processus de migmatisation peut être associé à la phase de collision entre la plaque bénino-nigériane et la marge orientale du craton ouest-africain. II serait donc pénécontemporain de la phase de granulitisation dans les massifs de la zone de suture (Affaton et al., 2000 ; Agbossoumondé et al., 2004). La signature structurale de cette ultime étape de convergence $(\mathrm{Dn})$ correspond à la foliation $\mathrm{Sn}$ qui se retrouve masquée ou quasi oblitérée au cours de la phase subséquente $D n+1$. Ainsi, cette marque transparait discrètement, avec une paragenèse quartzo-feldspathique entièrement réorientée et refondue dans la foliation principale $\mathrm{Sn+1}$. La phase de déformation $\mathrm{Dn}+1$ peut se définir comme un épisode de structuration majeure du complexe de Nikki. Elle est matérialisée par la foliation principale $\mathrm{Sn}+1$ associée à une forte linéation minérale ou d'étirement $L n+1$. Les empreintes syn-Dn+1 sont soulignées par une paragenèse à hornblende verte pouvant traduire une rétromorphose à faciès amphibolite. La foliation $\mathrm{Sn}+1$ est reprise par la phase de déformation $D n+2$ correspondant essentiellement au développement d'une grossière schistosité de flux $\mathrm{Sn}+2$ et surtout d'une famille de plans de cisaillement dextre $\mathrm{Cn}+2$. Ces plans $\mathrm{Sn}+2$ et $\mathrm{Cn}+2$ sont généralement soulignés par une recristallisation de biotite permettant d'associer à la phase $\mathrm{Dn}+2$ un second épisode de rétromorphose.

Le plan de cisaillement senestre $\mathrm{Cn}+3$ est attribué à la phase $\mathrm{Dn}+3$. Cette structure tardive est non pénétrative et soulignée par une paragenèse à fins cristaux de quartz, chlorite et magnétite permettant de lui associer une rétromorphose à faciès schiste vert. Dans l'ensemble des roches du complexe de Nikki, la rétromorphose syn-Dn+3 est accompagnée d'une recristallisation d'apparence post-tectonique représentée par des porphyroblastes d'épidote, de muscovite, de magnétite et de sphène. Cette dernière 
recristallisation serait liée aux effets thermiques de la mise en place des plutons granitiques plus ou moins tardifs (Castaing et al., 1993). Elle semble donc clôturer l'évolution métamorphique des ensembles lithologiques du complexe de Nikki.

A la fin de la phase de déformation $D n+3$, les matériaux granito-migmatitiques de Nikki ont enregistré un premier épisode de fracturation matérialisé par un système de décrochements indiquant un axe majeur de compression $\sigma 1 \mathrm{~N} 100$ à N110 (fig. $7 a$ et b). Les marqueurs du second épisode de fracturation, attribué à la phase de déformation $\mathrm{Dn}+4$ ne sont représentés que par des décrochements tardifs résultant d'une compression à axe majeur o1 SE-NW à SSE-NNW (fig. $7 c$ et d).

\begin{tabular}{|c|c|c|c|c|c|c|c|c|}
\hline & \multirow{2}{*}{ Site } & \multicolumn{2}{|c|}{ Coordonnées } & \multirow{2}{*}{$\mathrm{Rt}$} & \multirow{2}{*}{$\mathrm{Np}$} & \multirow{2}{*}{$\begin{array}{c}\sigma 1 \\
(\mathrm{D}-\mathrm{P})\end{array}$} & \multirow{2}{*}{$\begin{array}{c}\sigma 2 \\
(D-P)\end{array}$} & \multirow{2}{*}{$\begin{array}{c}\sigma 3 \\
(\mathrm{D}-\mathrm{P})\end{array}$} \\
\hline & & Latitude & Longitude & & & & & \\
\hline 1 & Serikali & N09 $54^{\prime} 14.9^{\prime \prime}$ & $\mathrm{E}^{\circ} 3^{\circ} 03^{\prime} 03.3^{\prime \prime}$ & $\mathrm{Gr}$ & 23 & $137-05$ & $235-61$ & $44-29$ \\
\hline 2 & Est-Nikki & N09 $55^{\prime} 36.6^{\prime \prime}$ & $\mathrm{E} 03^{\circ} 13^{\prime} 23.2^{\prime \prime}$ & OG & 18 & $99-07$ & $244-82$ & $09-05$ \\
\hline 3 & $\begin{array}{c}\text { Ouest- } \\
\text { Tchikandu }\end{array}$ & N09 $52^{\prime} 00.6^{\prime \prime}$ & E0320'31.6" & M & 33 & $151-01$ & $267-87$ & $61-02$ \\
\hline 4 & NE- & N09 $36^{\prime} 10.4^{\prime \prime}$ & $\mathrm{E} 02^{\circ} 57^{\prime} 53.8^{\prime \prime}$ & GM & 13 & $106-00$ & $14-77$ & 196-13 \\
\hline & & & & & 20 & $337-03$ & $74-67$ & $246-23$ \\
\hline 5 & $\begin{array}{c}\text { Sud- } \\
\text { Parakou }\end{array}$ & N09 $17^{\circ} 11.7^{\prime \prime}$ & $\mathrm{E} 02^{\circ} 34^{\prime} 51.2^{\prime \prime}$ & $\mathrm{Gr}$ & 12 & $103-03$ & $249-86$ & $13-02$ \\
\hline
\end{tabular}

Tableau 1 : synthèse des résultats de l'analyse des plans de décrochement relevés sur les sites faillés étudiés dans le complexe granito-migmatitique de Nikki. Le tableau résume, pour chaque site, les coordonnées géographiques, le type de roche $(\mathrm{Rt})$ fracturée $(\mathrm{Gr}=$ granite, $\mathrm{OG}=$ orthogneiss, $\mathrm{GM}=$ gneiss migmatitiques, $M=$ migmatites), le nombre de plans (Np) et les direction et plongement (D-P) des axes de contraintes principales $(\sigma 1, \sigma 2, \sigma 3)$ obtenus. 


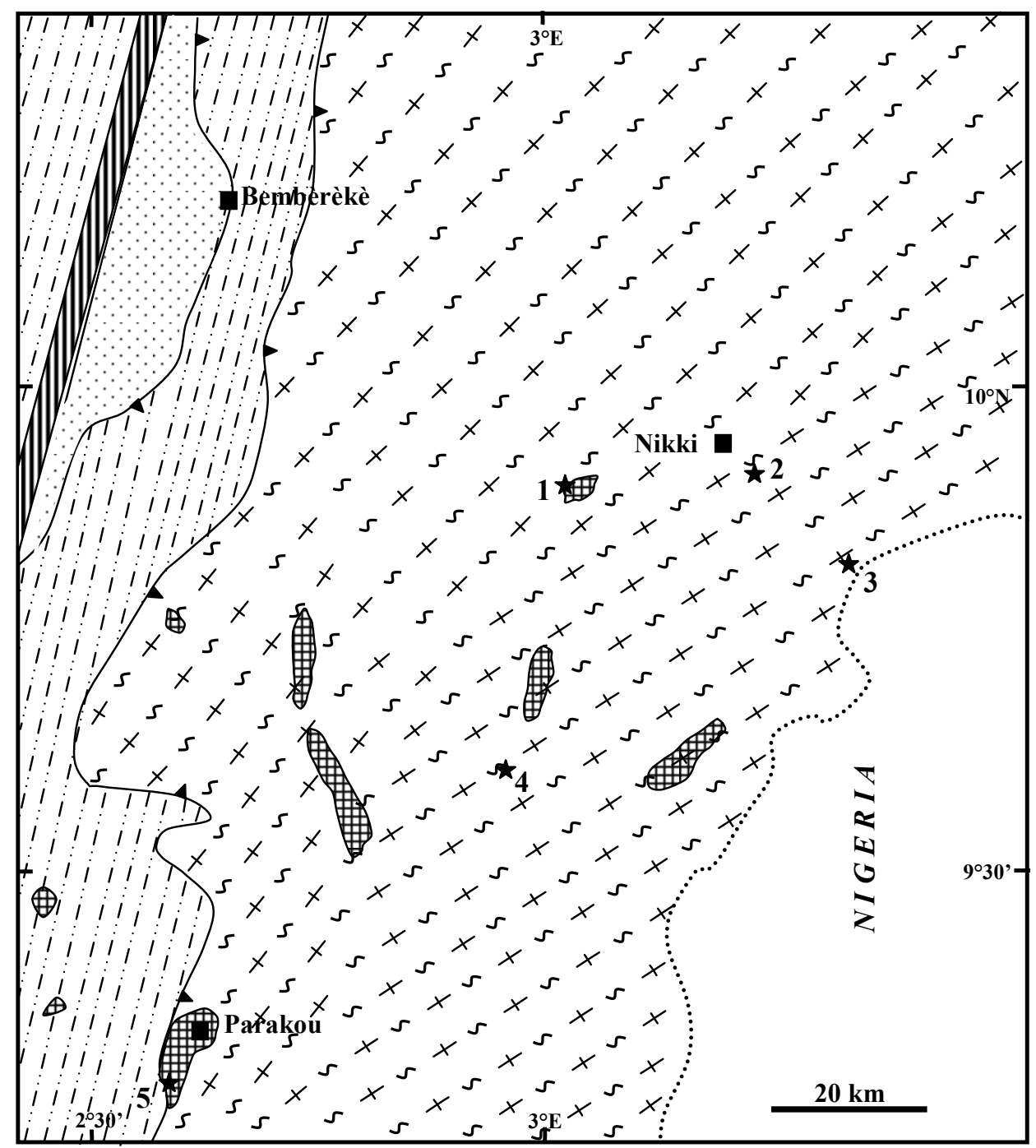

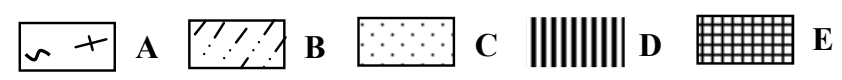

Fig. 2 : Esquisse de carte géologique du secteur de Nikki, avec situation des sites faillés étudiés $(1=$ Serekali, $2=$ Est-Nikki, 3 = Ouest-Tchikandu, 4 = NE-Parakou, 5 = Sud-Parakou) : A - unité du complexe granito-migmatitique de Nikki, B - unité du complexe de la zone axiale de la « Faille de Kandi », incluant les quartzites micacés à disthène de Bembèrèkè $(C)$ et les faciès mylonitiques $(D)$ de la zone de cisaillement ("Faille de Kandi »), E - intrusions granitiques. 

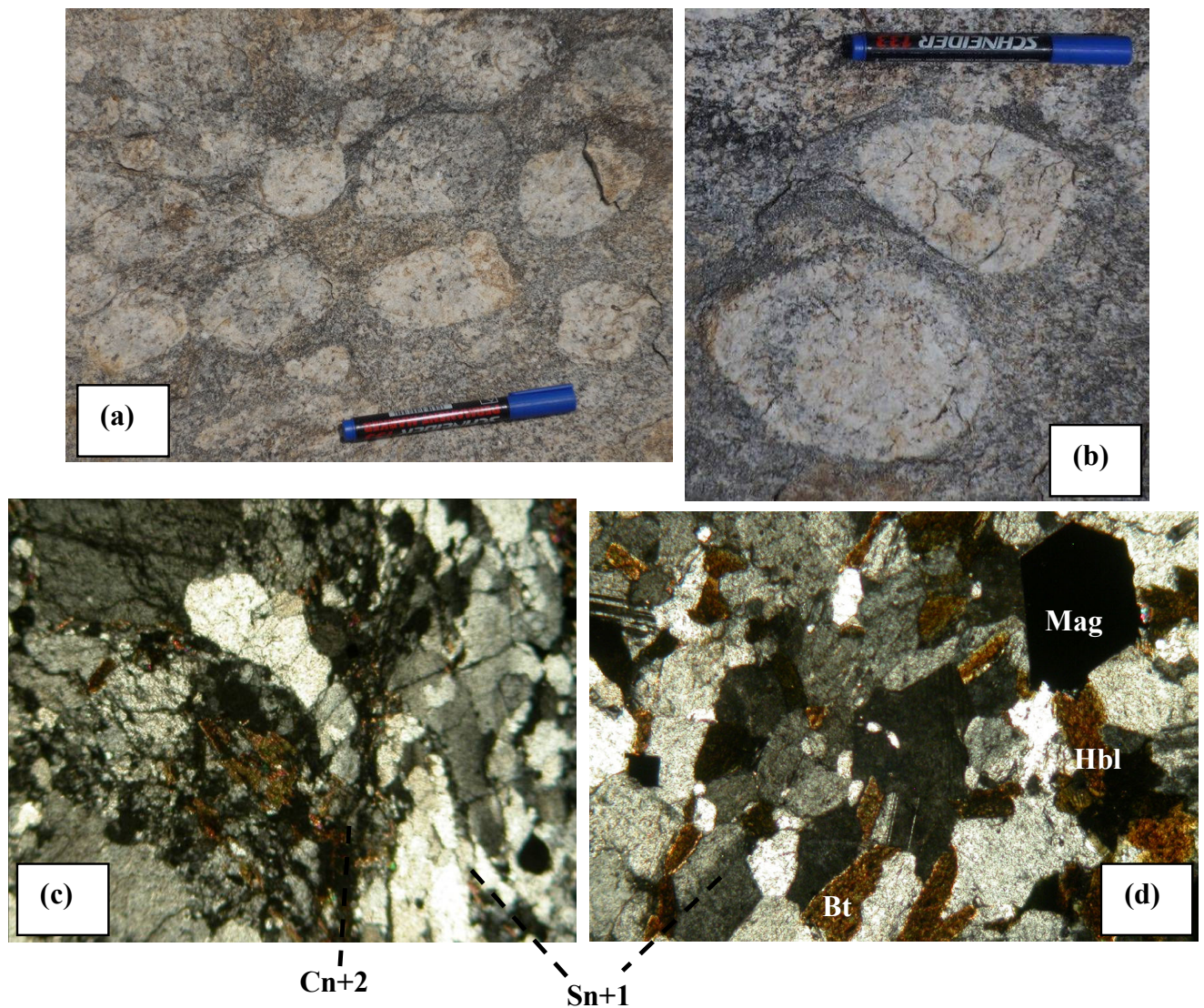

Fig. 3 : Caractéristiques structurales et microstructurales des faciès orthogneissiques du complexe de Nikki (a) orthogneiss à structure orbiculaire, avec des porphyroblastes centimétriques à décimétriques de feldspaths plus ou moins étirés dans la direction NE-SW ; (b) - détail sur des porphyroblastes montrant la structure interne à couronne de recristallisation; (c) et (d) - microtextures des orthogneiss montrant la foliation principale $(S n+1)$ recoupée par le plan de cisaillement dextre $(\mathrm{Cn}+2)$. 

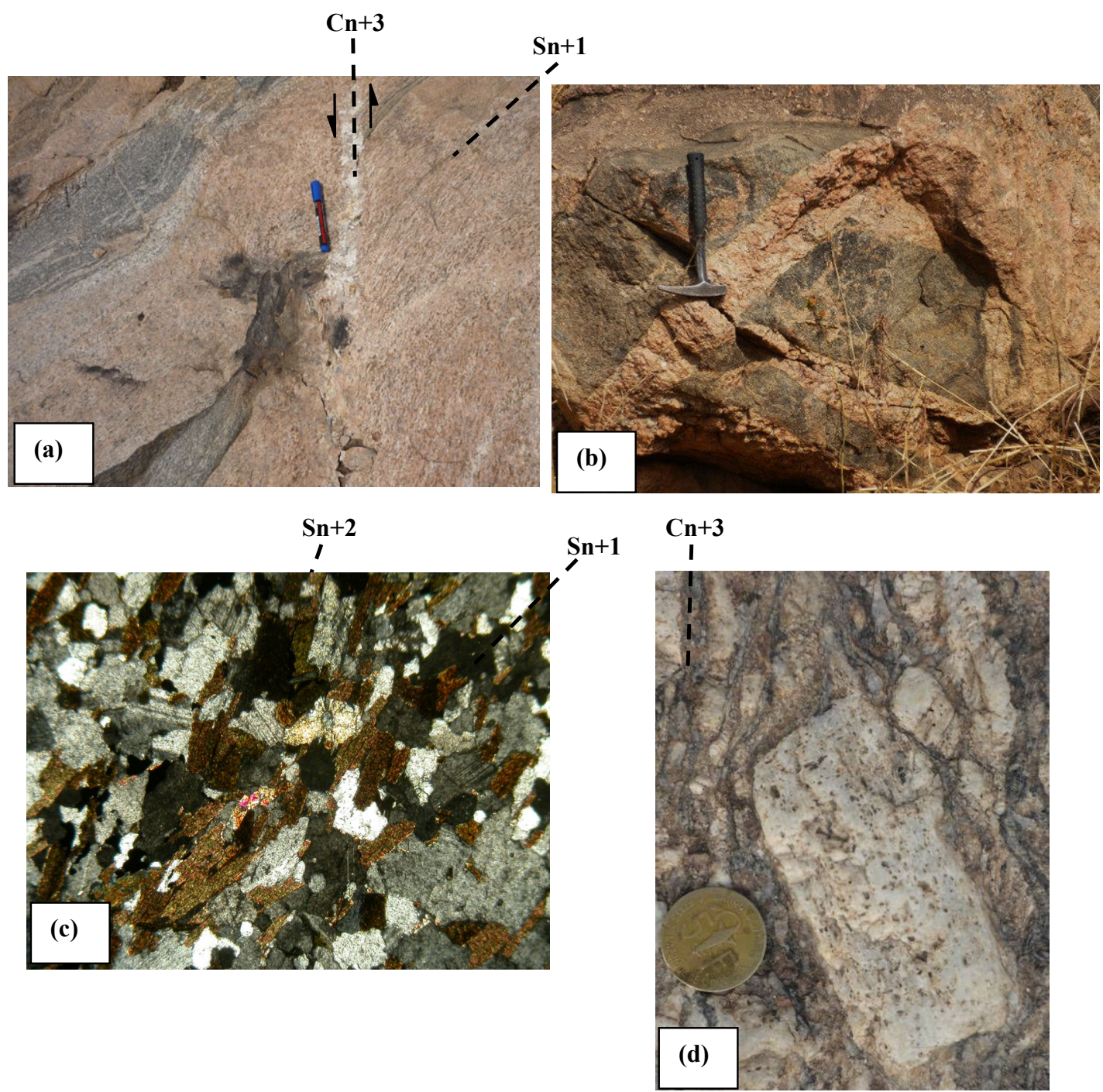

Fig. 4 : Caractéristiques pétrostructurales des migmatites et granites orientés du complexe de Nikki (a) - association d'orthogneiss granodioritique sombre et de métagranite rose structurée par la foliation $\mathrm{Sn+1}$ (N70) recoupée par le plan de cisaillement senestre (N20) ; (b) - association d'enclaves de gneiss et de métapegmatites à tourmaline ; (c) microtexture d'orthogneiss granodioritique montrant la superposition des foliations ou schistosités de flux $\mathrm{Sn+1}$ et $\mathrm{Sn}+2$; (d) - structure du granite de Parakou, avec des porphyroblastes centimétriques de feldspaths orientés et un plan de cisaillement senestre $\mathrm{Cn}+3$ de direction N35. 


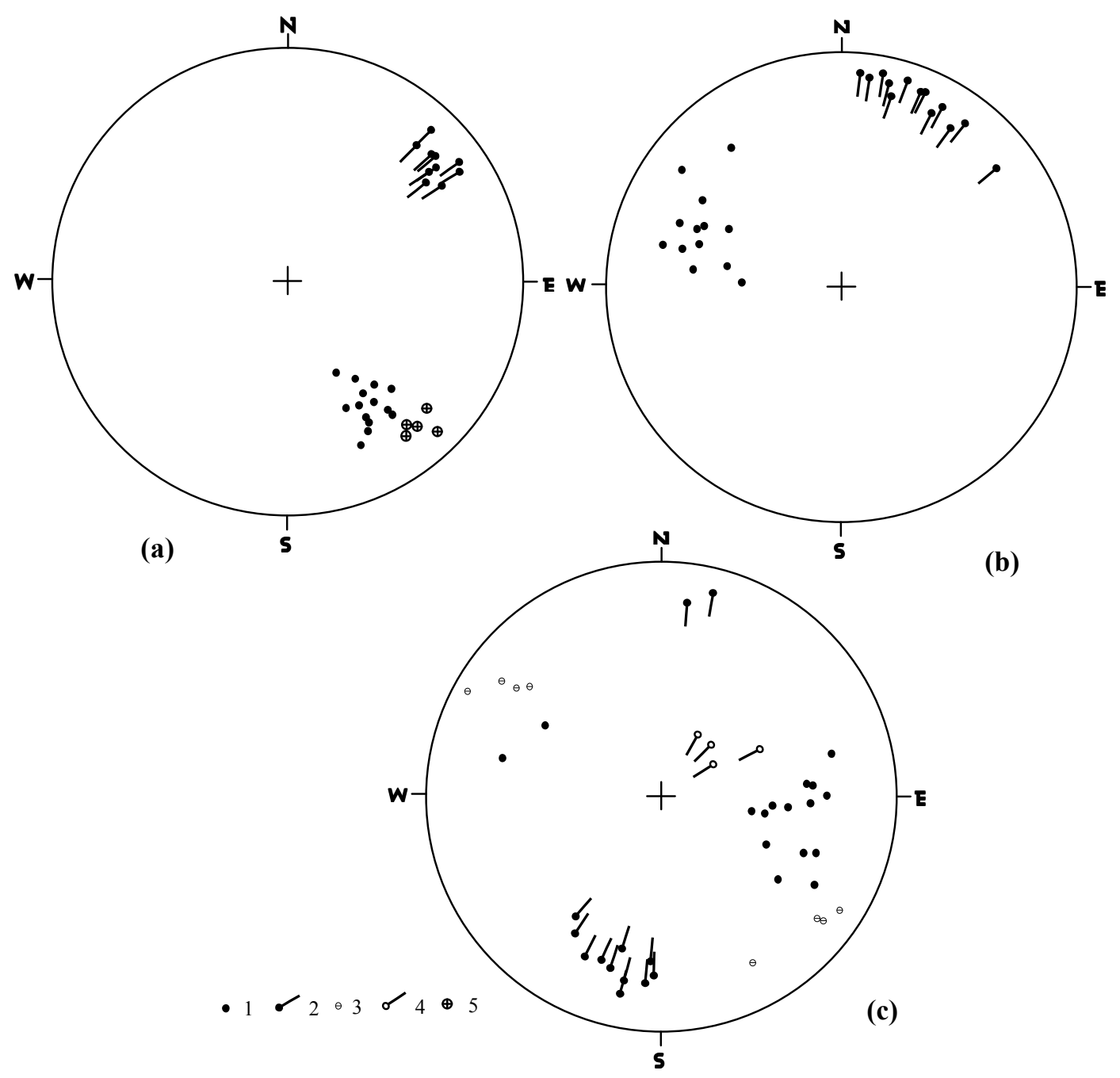

Fig. 5 : Stéréogrammes de synthèse des éléments structuraux relevés sur les affleurements orthogneissiques du complexe de Nikki. (a) - site Est-Nikki (N0955'36.6" - E0313'23.2"), (b) - site NE-Parakou (N09'36'10.4" -

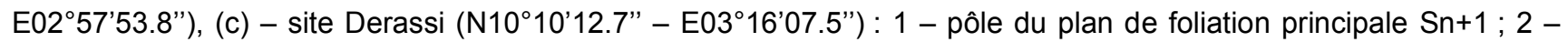
pôle de linéation minérale ou d'étirement $L n+1 ; 3$ - pôle du plan de cisaillement dextre $C n+2 ; 4$ - pôle d'axe de pli d'entraînement $\mathrm{Pn}+2$; 5 - pôle du plan de cisaillement senestre $\mathrm{Cn+3}$. 

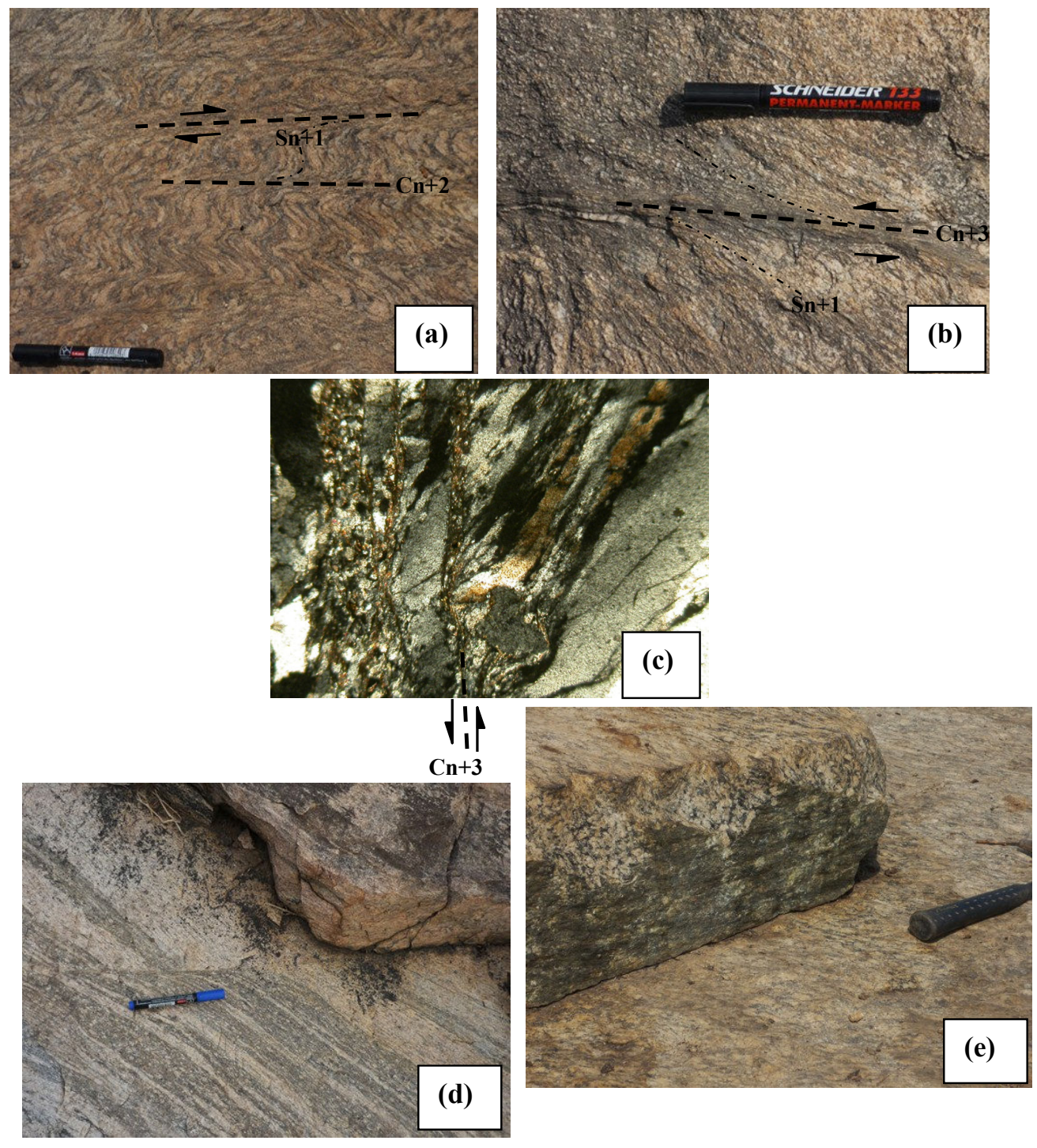

Fig. 6 : Principaux marqueurs de déformation panafricaine dans les orthogneiss et les migmatites du complexe de Nikki: (a) - plan de cisaillement dextre $(\mathrm{Cn}+2)$ N35 à N45 pénétratif, débitant la roche en « microlithons », avec des microplis d'entraînement dessinés par la foliation $\mathrm{Sn+1}$; (b) - plan de cisaillement senestre $(C n+3)$ N15 superposé au plan $\mathrm{Cn}+2$; (c) - microphotographie montrant la texture mylonitique du faciès soulignant le plan $\mathrm{Cn}+3$ en (b) ; (d) et (e) - miroirs de décrochements senestres syn-Dn+3 avec des recristallisations de quartz (d) et de biotite (e). 


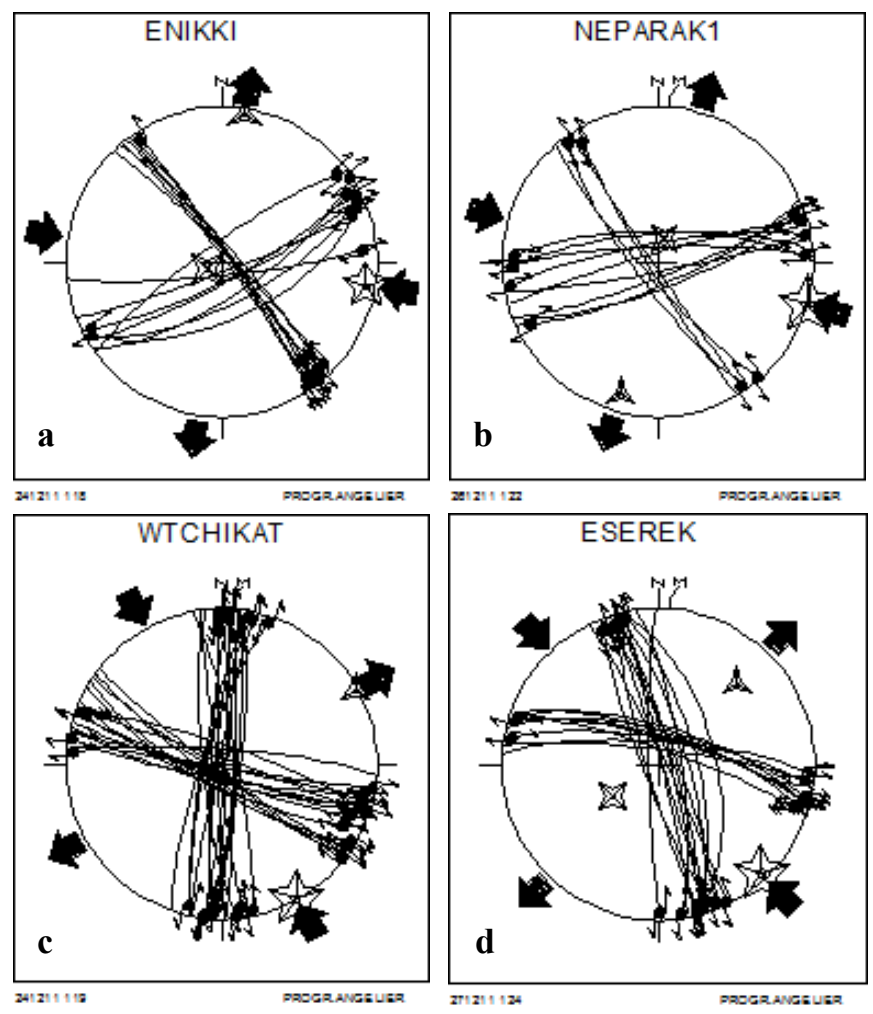

Fig. 7 : Stéréogrammes d'analyse des plans striés matérialisant les phases de déformation $\mathrm{Dn}+3(\mathrm{a}-$ site Est-Nikki et b - site NE-Parakou) et Dn+4 (c - site Ouest-Tchikandu et d - site Serekali). Les étoiles représentent les pôles des axes des contraintes principales $\sigma 1$ (étoile à 5 branches), $\sigma 2$ (étoile à 4 branches) et $\sigma 3$ (étoile à 3 branches)

\section{CONCLUSION}

Grâce à cette analyse des marqueurs de déformation, on peut décomposer en cinq phases la tectogenèse panafricaine enregistrée par le complexe granito-migmatitique de Nikki. La remobilisation panafricaine débute avec une large migmatisation probablement pénécontemporaine de la phase de collision Dn datée de 650 - 610 Ma (Affaton et al., 2000 ; Kalsbeek et al., 2012). Cette phase Dn est associée à la foliation $\mathrm{Sn}$ quasiment oblitérée par l'épisode majeure $\mathrm{Dn}+1$. La foliation principale $\mathrm{Sn+1}$ correspond à une parfaite transposition du plan $\mathrm{Sn}$ et à la refonte des paragenèses syn-Dn dans des conditions $\mathrm{du}$ faciès amphibolite. La phase $\mathrm{Dn}+2$ est particulièrement matérialisée par le plan de cisaillement dextre $\mathrm{Cn}+2$. II convient d'associer la généralisation de ce plan à l'épisode de développement du couloir majeur de cisaillement de la «Faille de Kandi ». La déformation $\mathrm{Dn}+3$ se traduit par la superposition d'un plan subméridien de cisaillement senestre $(\mathrm{Cn}+3)$. Elle se termine par un épisode de fracturation à l'origine d'un système de décrochements définissant un axe principal de compression ESE-WNW. Enfin, la dernière phase de tectogenèse panafricaine $(D n+4)$ ne se traduit que par un ensemble de décrochements tardifs matérialisant une paléocontrainte de compression majeure SE-NW à SSE-NNE.
La géométrie des axes $\sigma 1$ syn- $\mathrm{Dn}+3$ et syn$D n+4$ semble répondre à une rotation horaire de 40 à $50^{\circ}$ entre les axes de compression majeure au cours des deux dernières phases de l'édification de la chaîne panafricaine des Dahomeyides (Tairou et al., 2007 ; 2012). C'est cette rotation qui explique la superposition du plan de cisaillement senestre tardif $\mathrm{Cn}+3$ aux structures pénétratives syn-Dn+1 et syn-Dn+2 et, plus particulièrement, au plan de cisaillement dextre $\mathrm{C} n+2$. Des relevés complémentaires permettront de mieux contraindre la superposition des paléocontraintes panafricaine dans le NE-Bénin.

\section{RÉFÉRENCES}

Abdelsalam M.G., Liégeois J.-P., Stern R. J., 2002. The Saharan Metacraton. Jour. of Afr. Earth Sci., 34, pp. $119-136$.

Affaton P., 1990. Le basin des Volta (Afrique de 1'Ouest) : une marge passive d'âge Protérozoïque supérieur, tectonisée au Panafricain (600 $\pm 50 \mathrm{Ma})$. Edit. ORSTOM, Collection Etudes et Theses, Paris, $500 \mathrm{p}$.

Affaton P., Lassere J. L., Lawson L. T., Vincent P. L., 1978. Notice explicative des cartes géologiques à $1 / 200000$ de la République du Togo et 
de la République populaire du Bénin entre les $9^{\mathrm{e}}$ et $10^{\mathrm{e}}$ de latitude Nord (feuille BassariDjougou et feuille Parakou - Nikki). Rapp. Bur. Nat. Rech. Min. du Togo (B.N.R.M.) Bur. Rech. Géol. Min. Fr. (B.R.G.M.) et Off. Béninois Mines (OBEMINES), 70 p.

Affaton P., Sougy J., Trompette R., 1980. The Tectono-Stratigraphic Relationships between the Upper Precambrian and Lower Paleozoic Volta Basin and the Pan-African Dahomeyide Orogenic Belt (West Africa). Amer. Jour. Sci., 280 , pp. 224 - 248.

Affaton P., Rahaman M. A., Trompette R., Sougy J., 1991. The Dahomeyide Orogen : Tectonothermal Evolution and Relationships with the Volta Basin. In Dallmayer and Lécorché (Eds): The West-African Orogen and Circum Atlantic Correlatives. Projet 233. 1CGP, IUGS, UNESCO, pp 107 - 122.

Affaton P., Kröner A., Seddoh K. F. 2000. Pan African Granulites Formation in the Kabye of Northen Togo (West Africa): $\mathrm{Pb}-\mathrm{Pb}$ zircon ages. Int. Jour. Earth Sci. 88, pp. 778 - 790.

Agbossoumonde Y., 1998. Les complexes ultrabasiques de la chaîne panafricaine au Togo (Axe Agou Atakpamé, Sud-Togo). Etude pétrographique, minéralogique et géochimique. Thèse Doct. Lab. Géol. Pétro. Univ. Jean Monnet St. Etienne Fr., 306 p.

Agbossoumonde Y., Guillot S., Ménot R.-P., 2004. PanAfrican Subduction - Collision Envent Evidence by Hight-P Coronas in Metanorites from the Agou - Massif (Southern Togo). Precambrian Research, 135, pp. $1-21$.

Agbossoumonde Y., Ménot R.-P., Paquette J. L., Guillot S., Yessoufou S., Perrache C., 2007. Petrological and Geochronological Constraints on the Origin of the Palimé-Amlamé Granitoids (South Togo, West Africa): A Segment of the West African Craton Paleoproterozoic Margin Reactivated during the Pan African Collision. Gondwana Research, 12 (4), pp. 476-488.

Angelier J., 1990. Tectonique cassante et néotectonique. An. Soc. Géol. Belgique, (112) 2, pp. 283-307.

Attoh K., Dallmeyer R. D., Affaton P., 1997. Chronology of Nappe Assembly in the Pan-African Dahomeyide Orogen, West Africa: Evidence from ${ }^{40} \mathrm{Ar} /{ }^{39} \mathrm{Ar}$ Mineral Ages. Precambrian research, 82, pp. $153-171$.

Attoh K., Corfu F., Nude M. P., 2007. U-Pb Zircon Age of Deformed Carbonatite and Alkaline Rocks in the Pan-African Dahomeyide Suture Zone, West Africa. Precambrian Research. 155, pp. 251260.
Attoh K., Nude P. M., 2008. Tectonic Significance of Carbonatites and Ultrahigh-Pressure Rocks in the Pan-African Dahomeyide Suture Zone, Southeastern Ghana. In Ennih N. and Liégeois J.-P. (Eds): The Boundaries of the West African Craton. Geol. Soc. London, Special Publications, 297, 217-231.

Caby R., 1989. Precambrian Terranes of Benin Nigeria and Northeast Brazil and the Late Protererozoic South Atlantic Fit. Geol. Soc. Am, Special Paper 230, pp. 145 - 158.

Caen-Vachette M., 1975. Age pan-africain des granites de Sinendé, Save et Fita (Dahomey). C. R. Acad. Sci., Paris, pp. 1793 - 1795.

Caen-Vachette M., Pinto K.J.M., Roques M., 1979. Plutons éburnéens et métamorphisme dans le socle cristallin de la chaîne panafricaine au Togo et au Bénin. Rev. Géol. Dyn. Géogr. Phys. 21 (5), p. 351.

Castaing C., Triboulet C., Feybesse J. L., Chevremont P., 1993. Tectono-Metamorphic Evolution of Ghana, Togo, and Benin in the Light of the PanAfrican/Brasiliano Orogeny. Tectonophysics, 218, pp. $323-342$.

Duclaux, G., Ménot, R. P., Guillot, S., Agbossoumondé, Y., Hilaret, N., 2006. The Mafic Complex of the Kabyé Massif (North Togo and North Benin): Evidence of a PanAfrican Granulitic Continental Arc Root. Precambrian research. 151, pp. 101-118.

Kalsbeek F., Affaton P., Ekwueme B.,Frei R., Thrane K., 2012. Geochronology of Granitoid and Metasedimentary Rocks from Togo and Benin, West Africa: Comparisons with NE Brazil. Precambrian research 196-197, pp. 218-233.

Ngako V., 1999. Les déformations continentales panafricaines en Afrique Centrale. Résultat d'un poinçonnement de type hymalayen. Thèse d'Etat, Univ. Yaoundé I, $301 \mathrm{p}$.

Rahaman M. A., Ukpong E. E., Azmatullah M., 1988. Geology of Parts of the Oban Massif, Southeastern Nigeria. Jour. Min. Geol. 15 (1), pp. $38-56$.

Sabi B. E., 2007. Etude pétrologique et structurale du Massif Kabyè, Nord-Togo. Thèse Doctorat, Univ. Lomé, $256 \mathrm{p}$.

Simpara N., 1978. Etude géologique et structurale des unités externes de la chaîne panafricaine (600 Ma) des Dahomeyides dans la région de Bassar (Togo). Thèse $3^{\mathrm{e}}$ cycle, Univ. AixMarseille III, $164 \mathrm{p}$.

Simpara N., Sougy J., Trompette R., 1985. Lithostratigraphie et structure du Buem, unité externe de la chaîne panafricaine des 
Dahomeyides de la région de Bassar (Togo). Jour. of Afr. Earth Sci., 3 (4), pp. 479 - 486.

Tairou M. S., 2006. La tectonique tangentielle panafricaine au Nord-Togo. Thèse Doctorat, Univ. Lomé, 135, 401 p.

Tairou M. S., Affaton P., Gelard J.-P., Aite R. Sabi B. E., 2007. Panafrican Brittle Deformation and Paleostress Superposition in the Northern Togo (West Africa). C. R. Géosciences 339, pp. 849-857.
Tairou M. S., Affaton P., Sabi B. E., Seddoh K. F., 2009. Tectono-Metamorphic Evolution of the Mo and Kara-Niamtougou Orthogneissic Suites, Northern Togo. Global Jour.of Geological Sciences, 7 (2), pp. 93-100.

Tairou M.S., Affaton P., Anum S., 2012. Pan-African Paleostresses and Reactivation of the Eburnean Basement Complex in Southeast Ghana (West Africa). Journal of Geological Research, Vol. 2012, pp. 1-15. 